\title{
The Cash-Point (ATM) 'Problem'
}

\author{
Tim Denvir ${ }^{1}$, José Oliveira ${ }^{2}$ and Nico Plat ${ }^{3}$ \\ ${ }^{1}$ Translimina Ltd., London, UK \\ ${ }^{2}$ Universidade do Minho, Braga, Portugal \\ ${ }^{3}$ West Consulting, Delft, The Netherlands
}

\begin{abstract}
This paper provides a description and summary of the solutions submitted to a competition in formal specification, which was held during FM'99 in Toulouse, September 1999.
\end{abstract}

Keywords: Cash-point (ATM) problem; Competition; FM'99, Formal methods, Tools exhibition

\section{Introduction}

The FM'99 Congress [FM'99, FM'99 FACJ], organised by the association Formal Methods Europe [FME], was held in Toulouse in September 1999. Selected technical papers from FM'99 appear in special issues of three different journals towards the end of 2000: IEEE Transactions on Software Engineering, Formal Methods in System Design, and Formal Aspects of Computing [FM'99 FACJ]. FM'99 included a formal methods tools exhibition. Exhibitors were invited to take part in a competition by applying their formalism and tool to a particular problem. There were fifteen entries to this competition, of which eight are summarised immediately after this paper. The competition was organised by Tim Denvir and Nico Plat. The jury consisted of three prominent FME members: Göran Anger, Cliff Jones and José Oliveira.

The task, which was the basis for the competition, did not present any special technical difficulty in the sense of being an 'unsolved problem'. This was deliberate; the intention was to provide a practical problem that had the requirements of security, concurrency, need for correct behaviour under various hazards and so forth that are typical of serious real-life applications. We wished to observe how the different formalisms and tools could be used to respond to such demands. As a result, this paper does not claim to display specific theoretical advances, but provides a picture of the practical performance of some formal methods tools and underlying formalisms, which may be of interest to the formal methods community.

Exhibitors at FM'99 were invited to specify the service described below using their formalism, and to use their tool to process that specification. Proofs of properties related to security of transactions would be especially interesting, and those entering the competition were asked to explain what benefits result from using facilities of their tool. They were asked to make an informal 20-minute presentation of their solutions at their exhibition stands.

Correspondence and offprint requests to: T. Denvir, Translimina Ltd., 55a Compton Road, Winchmore Hill, London N21 3NU, UK. Email: t-denvir@dircon.co.uk 


\section{The Problem}

The problem was taken from a previous workshop on 'The Analysis of Concurrent Systems' held in Cambridge in September 1983. The description of the task was updated slightly so as to reflect advances in technology and understanding since then. In the 1983 workshop, reported in LNCS 207 [Den86], the organisers formulated ten problems in formal specification and invited some twenty-six leading computer scientists to present solutions. There were thirty-three solutions in total, but none were provided for the 'ninth problem', although it did not present any special difficulty compared with the others. The competition for the FM'99 exhibitors was based on this ninth problem.

\subsection{The Service to be Specified}

There are many tills, which can access a central resource containing the detailed records of customers' bank accounts. Customers insert a card into the till and type in a PIN (Personal Identification Number), which is encoded by the till and compared with a code stored on the card.

After successfully identifying themselves to the system, customers may try to:

1. view the balance of their accounts;

2. make a withdrawal of cash;

3. ask for a statement of their account to be sent by post.

Information on accounts is held in a central database and may be unavailable. In this case (1) above may not be possible. If the database is available, any amount up to the total in the account may be withdrawn, subject to a fixed daily limit on withdrawals. This means that the amount withdrawn within the day must be stored on the card.

'Illegal' cards are kept by the till.

The solution should consider feasible implementation architectures. These are likely to include a data line between each till and the central database. For example, transactions at a till may be recorded locally and sent in a queue down the line to the central database.

\subsection{Concurrency, Time Constraints}

Candidates are asked to show that their solutions allow for concurrent access to the database from two or more different tills. It is important that once a user has initiated a transaction, the transaction is completed at least eventually, and preferably within some real time constraint. Candidates should also consider how to handle concurrent attempts from two cardholders who are authorised to use the same account.

\subsection{Security, Reliability}

Clearly, for this sort of application, it is important to minimise the possibility of stolen cards being used to gain access to an account, and to ensure that implementations of the system are robust against various hazards such as poor transmission between a till and the central database. Any way in which solutions can address such issues is particularly welcome.

\subsection{Proofs}

Any proofs or demonstrations (e.g. by animation or model checking) of concurrent accessibility, temporal properties and security and reliability aspects outlined above are especially welcomed, as are proofs of any provided reification. 


\subsection{Abstraction}

Notwithstanding the above, the specifications provided should be reasonably abstract. A feasible design for an implementation could be presented as a first-stage reification of the abstract specification.

\section{The solutions}

The FM'99 tools exhibition enrolled 35 stands. Twelve of these became involved in the cash-point competition and presented a solution to the problem at the symposium. Eight of these submitted a report about of their solution:

\begin{tabular}{clccl}
\hline Stand & Tool(s) & Prize & Report & Institution(s) \\
\hline 01 & VDM ToOLS & - & $*$ & IFAD (Denmark) \\
02 & UPPAAL & - & - & Aalborg Univ. (Denmark) \& Uppsala Univ. (Sweden) \\
03 & STeP & 2 nd & $*$ & CSD, Stanford (USA) \\
04 & MoBY/PLC & - & $*$ & Univ. Oldenburg (Germany) \\
05 & NUT & - & $*$ & Tallinn Tech. Univ. (Estonia) \\
06 & AUTOFocus with Quest & 1 st & $*$ & TU Munich (Germany) \\
07 & LPV-Technology & - & - & GREYC - Univ. of Caen (France) \\
08 & ObjectGEODE \& SCADE & - & - & VERILOG (France) \\
09 & LSR \& VERIMAG & - & $*$ & Lab. LSR-IMAG (France) \\
10 & DisCo Tool & - & $*$ & S.S.Lab, Tampere Univ. (Finland) \\
11 & ESTEREL STUDIO & 3 rd & $*$ & Esterel Technologies (France) \\
12 & B-Tools & - & - & B-Core (UK) \\
\hline
\end{tabular}

Below we present a brief comparative analysis of eight such solutions.

\subsection{Preamble}

Formal specification techniques can be broadly divided in two rough classes: model-oriented and processoriented techniques. Model-oriented techniques produce pre/post-operation specifications on top of settheoretic models of data and/or local state. They are particularly useful in addressing static, data-intensive systems. By contrast, process-oriented techniques concentrate on the specification of the dynamic aspects of systems. Data and state structures are regarded as less important than the interaction among the dynamic components of the target system. They are particularly useful in describing the behaviour of communicationintensive systems. Tools based on techniques of both classes were present in the competition.

\subsection{General Comments}

Most of the requirements of the cash-point (ATM) problem were taken into account by all solutions submitted, although in a variable degree of formality. This can be partly explained by the nature of the problem itself, which suits the system 'behaviourist' better than the 'data modeller'. In general, the system's architecture proposed at the abstract level consists of a central database networked with more than one till. Some solutions make the channels among the networked components explicit in order to specify security and reliability issues (e.g. transmission errors). A group of solutions (notably MoBY/PLC) go further into specifying real-time properties of the system. Others take advantage of a system specification closed with its environment in such a way that events like losing a card or finding a lost card can also be expressed. The only requisite which is apparently ignored in all the proposed solutions (or, at least, not mentioned) is the handling of concurrent attempts from two cardholders who are authorised to use the same account. 


\subsection{Tool Support}

\subsubsection{Animation/Simulation}

Concerning tool support, most solutions report benefits from the particular animation or simulation facilities available in the toolset. The usefulness of these facilities as a means to obtain feedback in the early modelling phases (rapid prototyping) is acknowledged in general. Some tools (e.g. VDM Tools and AutoFocus) incorporate test case generation facilities.

\subsubsection{Verification}

The requirements of the cash-point problem put special emphasis on automated verification of the concurrent, temporal properties and security (and reliability) aspects of the problem. Not all solutions address these concerns. Wherever they do, model checking is as a rule adopted as the verification technique and some tools actually integrate with independently developed model checkers. This brings about the issue of integration and complementarity.

\subsubsection{Integration and Complementarity}

The LSR \& VERIMAG and VDM TooLs solutions resort to UML object-oriented modelling and actually interface with Rational Rose, combining the complementary benefits of visual modelling (in UML) with precise modelling (in Z and VDM++ respectively). The VERIMAG approach actually extends this concern for complementarity to a multi-formalism approach, by mapping UML into orthogonal formalisms (Z, Lustre) and tools (RoZ, Z-eves, Lutess) in order to consider both the static and dynamic parts of specifications such as in the cash-point problem.

\subsubsection{Real Time}

The description of the problem mentions the time constraints which are in practice relevant to the operation of cash-points (e.g. by keeping a card after too long a delay). This aspect of the problem is very prominent in the Moby/PLC solution and also taken into account by others (e.g. NUT, STeP and EsTERel StUdio).

\subsubsection{Abstract Modelling}

The problem requirements state that the specifications provided should be reasonably abstract. This requirement is met in different ways by the provided solutions. A broad group of solutions (including DisCo, LSR \& VERIMAG, VDM ToOLS) resort to object-oriented modelling as a way of balancing the static/dynamic sides of the semantics of the problem. Concerning the modelling techniques, solutions often provide abstract data types for the static part (e.g. AutoFocus, VDM Tools) and/or state transition diagrams (or variants thereof) for the dynamic one (e.g. AutoFocus, Esterel Studio, STeP, Moby/Plc). The STeP solution, which is based on temporal specifications, is also interesting concerning parameterisation and modularisation.

\subsubsection{Reification}

Reification, understood as the process of stepwisely changing the level of abstraction of a solution down to the implementation level, is not explicitly provided in any of the reported solutions. However, some of these (notably EsTEREL STUDIO and VDM TOOLS) do take advantage of code-generation facilities (generating C/VHDL and Java respectively).

\subsubsection{Graphical Interfacing}

Most tools exhibit a graphical user interface in order to improve their usability. AutoFocus and EstereL STUDIO are particularly effective in this respect. VDM Tools and the LSR \& VERIMAG tools integrate with other toolsets (notably Rational Rose) and can thus take further advantage of foreign graphical modelling facilities (e.g. editors, browsers). 


\subsection{About the Winning Solution}

AutoFocus with Quest was regarded as the most comprehensive solution in the competition. It was chosen as the winning solution mostly because of its wide scope and ability to cope with almost all aspects of the cash-point problem.

AutoFocus's modelling technique is targetted at specifying complex, component-based distributed systems in a threefold way: its notation comprehends a functional part, a behaviour part and an interaction part, respectively modelled by DTDs (abstract datatype definitions), STDs (starte transition diagrams) and EETs (extended event traces). This makes it possible to integrate several validation techniques such as static typechecking (consistency checks) and model-checking. Safety-critical properties can be checked using SMV and SATO in a way which includes abstraction techniques to reduce complexity. Proof obligations can be verified using VSE. The report mentions several modelling errors in the cash-point problem which were corrected in this way, using test-cases based on negating desirable formal properties. The system exhibits an attractive graphical user interface which exploits the hierarchical structure of all description techniques in the tool.

\section{References}

[Den86] B. T. Denvir et al. (eds.): The Analysis of Concurrent Systems. LNCS 207, Springer-Verlag, 1986.

[FM'99] Formal methods. In World Congress on Formal Methods in the Development of Computing Systems, Toulouse, France, September 1999. Proceedings, Volumes I and II, J. M. Wing, J. Woodcock and J. Davies (eds.). LNCS 1708 and 1709 , Springer-Verlag, 1999.

[FM'99 FACJ] Reference to Formal Aspects of Computing, 12(3): 145-210, 2000.

[FME] http://www.fmeurope.org

Eight of the entries are published in the following pages. 